\title{
Advancing healthcare technology education and innovation in academia
}

\author{
A national network of medical technology incubators provides experiential training for the next generation of \\ medical entrepreneurs and enables creation of innovative technologies for pressing clinical needs.
}

T rainees at universities often have big ideas, motivation and the time needed to improve healthcare in meaningful ways. While there are a plethora of medical problems needing better solutions and trainees eager to engage, the lack of funding, mentorship and experience are considerable barriers to entry for student-innovators. Furthermore, the experiential, multidisciplinary and fluid nature of medical innovation is not conducive to the faculty-driven, classwork-based structure of traditional academic courses. In 2013, Sling Health, formerly known as IDEA Labs, started in St. Louis as a student-led medical technology incubator to overcome these challenges and enable trainees to go beyond learning the current state of healthcare to instead start advancing management ${ }^{1}$. Here we describe the multi-university, chapter-based structure and national impact of Sling Health over its first seven years. We highlight the importance of health technology education and innovation in academia, especially as COVID-19 disrupts so much of how we practice medicine.

\section{Student experience}

Sling Health is a student-led, grassroots initiative that brings together multidisciplinary students, physicians and mentors to develop innovative solutions to clinical challenges ${ }^{1}$. This program adopted the strengths of several leading biomedical design programs across the country ${ }^{2-4}$ and modified them into an easily accessible, low-cost, scalable model. Sling Health forms multidisciplinary teams around clinician- and patient-identified problems, then provides an ecosystem including mentorship, training materials, funding and prototyping facilities needed to advance medical device and software development. Operating on a volunteer-run model, Sling Health consists of chapters, each with a local student leadership team that works with a national Sling Health leadership core consisting of students from various chapters, as well as advisory boards consisting of clinicians, academic professors, healthcare leaders and venture capitalists who help shape the overall direction of the organization. Chapters each adapt the overall program framework described below to best fit their local entrepreneurial ecosystem.

Academic centers are a renewable source of skilled and motivated students training in various disciplines. Sling Health leverages this talent pool to bring together students from different schools (for example, medicine, engineering, business and law) that rarely have opportunities to work together in their respective educational programs. Each cycle, selected project leaders present interesting problem areas to program applicants. Project leaders and the executive boards then form teams around problems on the basis of mutual interests and complementary skills. In contrast to comparable programs ${ }^{5}$, Sling Health does not offer any credit or payment to students, which results in the self-selection of individuals genuinely interested and motivated by medical innovation and medical business development. Additionally, many chapters have negotiated agreements with their associated universities so Sling Health teams maintain $100 \%$ ownership of their intellectual property.

Throughout the year, the local and national executive boards work with physicians, allied health professionals and external partners such as the American Medical Association (AMA) to identify key clinical problems and collate a vetted clinical problem database. While some project leaders bring problems from personal experience, many use Sling Health's clinical problem database to identify interesting problem areas and clinical mentors. After teams form around problem areas of interest, students work with clinical and business advisors to hone the team's guiding problem statement on the basis of clinical need analysis, market analysis and patent literature review. This problem-centric approach allows teams to design solutions targeted to the clinical needs and enables teams to pivot to different solutions and plans more effectively than teams anchored to a particular technology.
Sling Health teams go through a three-phase education and development program as described previously ${ }^{1}$ (Fig. 1). This consists of the invention phase (needs assessment, market analysis, in silico design and iterative prototyping), the development phase (developing business plans and filing provisional patents), and the launch phase (creating a new company, seeking more funding and creating plans for their startup after Sling Health). Education is facilitated by a wiki-based curriculum curated by Sling Health, periodic design reviews for teams to get feedback from mentors and experts, and legal and funding support from national sponsors and local academic center resources. Each chapter also hosts a local Demo Day for teams to present prototypes, pitch to judges and outside investors, and compete for a chance to attend the National Demo Day.

Sling Health operates a multi-level structure consisting of a national board of directors, a network-wide executive board, and multiple chapter executive boards, guided by local and national strategic advisory boards, as well as hundreds of mentors providing direct assistance to teams. Student leaders at local chapters are attuned to needs, opportunities and challenges facing their peers on teams, enabling real-time adjustments to the program. The local and national boards have parallel structures that allow each member of local leadership teams to access relevant expertise from the national board and contemporaneous suggestions from their counterparts at other chapters to optimize chapter performance and, ultimately, team success.

There are practical limitations inherent to a volunteer-driven organization with yearly turnover that can threaten sustainability. To mitigate this risk, Sling Health continually refines a leadership pathway that facilitates institutional knowledge retention and accumulation. Leadership roles and responsibilities are closely aligned with members' career goals to provide experiential professional training and maintain a motivated leadership 

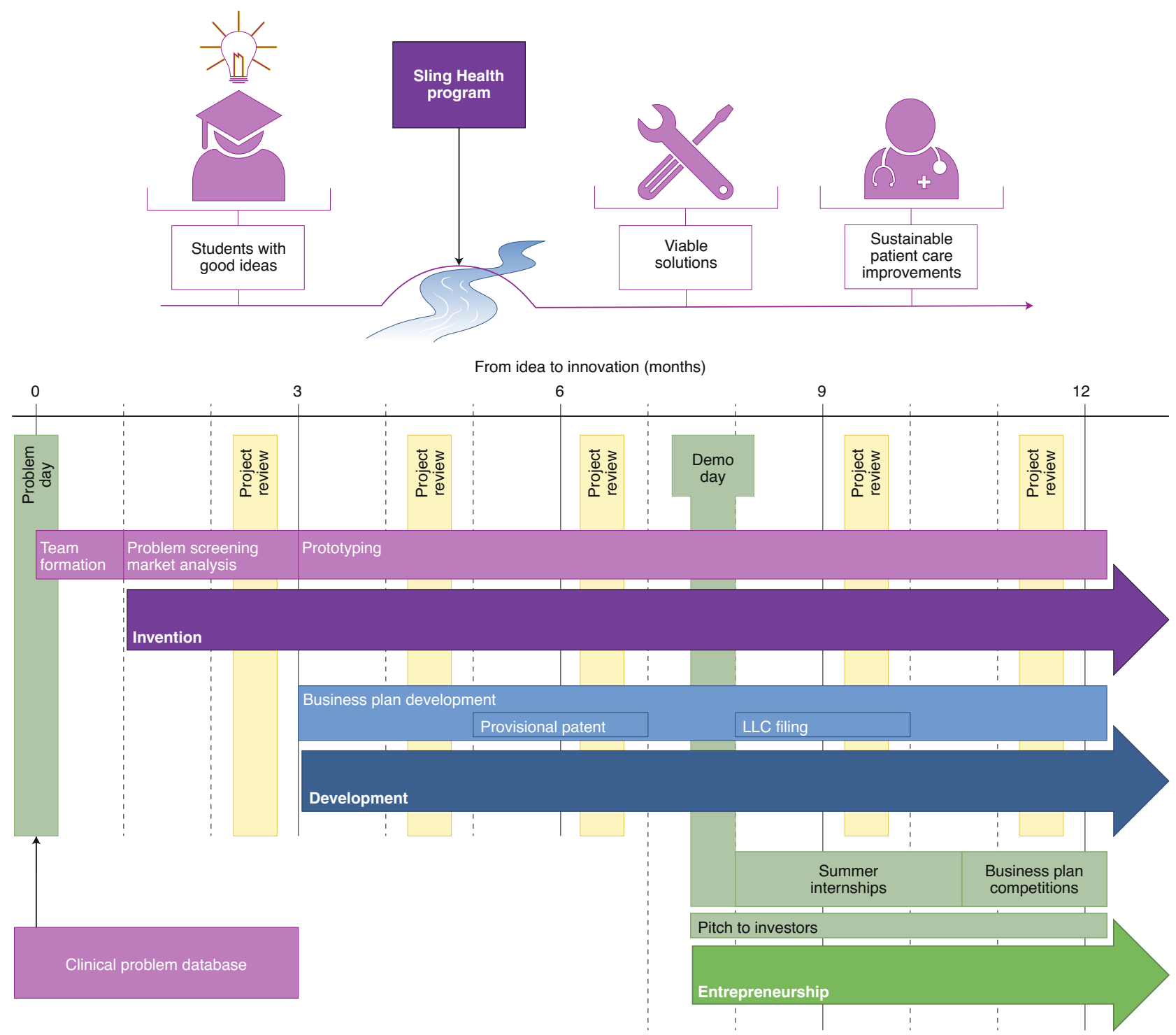

Fig. 1 | Sling Health's solution development pathway. Teams follow an entrepreneurial curriculum within the Sling Health program, leading teams from problem identification through to launching a startup or company.

base. Most participants choose to serve on executive boards for multiple years, while the advisory boards and the board of directors provide additional longitudinal oversight and continuity. Furthermore, executive transitional documents and project management technologies facilitate retention of institutional knowledge. This organizational structure has proven highly effective, sustainable and reproducible across chapters over the last seven years and across multiple transitions in national leadership.

\section{National network results}

Since 2015, Sling Health has rapidly grown as a national network with chapters spanning the country (Fig. 2), allowing leadership at each site to share knowledge, materials, infrastructure, operational costs and connections to the talent, mentors and investors best suited for each project. Sharing a suite of nationally managed back-end infrastructure including banking, accounting, legal, web hosting and IT resources reduces chapters' operational responsibilities and allows each chapter's volunteer leadership to focus on supporting teams and improving the local program. Sling Health's national scale enables far more students to gain experiential training in medical innovation (Fig. 3a) and allows all chapters to benefit from national partnerships and collaborations, as well as increased investor visibility, thus increasing chances of new technologies reaching patient care.
Sling Health actively works with trainees at potential new chapter sites to build on the local entrepreneurial ecosystem and bring together the necessary resources to enable medical technology development. Typical new chapters start with three to five executive board participants spanning medical, engineering and business training programs at undergraduate through postdoctoral levels. Student founders at new sites leverage national advisory support, existing chapter models, operational timelines, example presentations, outreach materials, a playbook of training materials and a full suite of national back-end infrastructure. This process allows new sites to learn from the experiences accrued throughout the network and pull together 


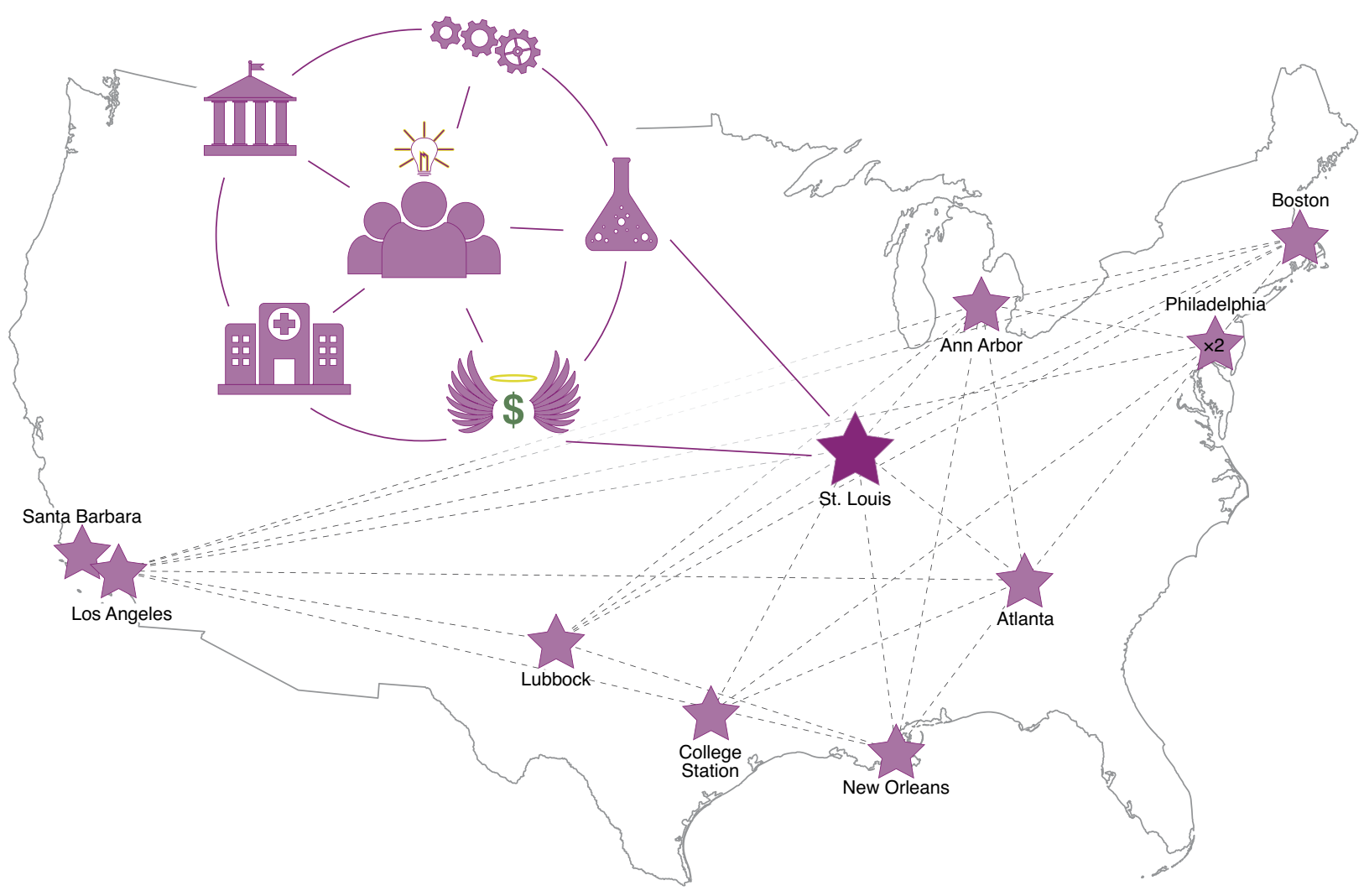

Fig. 2 | Sling Health's fit in an academic medical center ecosystem. Sling Health helps to coalesce medical, engineering, scientific, legal, and entrepreneurial or business resources available at universities into a medical entrepreneurial community to support student innovation. Sling Health further connects these separate communities at different institutions across the country into a cohesive network. There are currently 11 chapters involving 15 universities in the United States. Active chapter sites include St. Louis, MO; Boston, MA; Philadelphia, PA; Ann Arbor, MI; Atlanta, GA; College Station, TX; Lubbock, TX; New Orleans, LA; Los Angeles, CA; and Santa Barbara, CA.

the necessary local resources and support to develop a successful chapter much more quickly than 'reinventing the wheel' and starting new, independent programs at individual sites. As a result of this support, what took thousands of collective volunteer-hours to develop at the initial St. Louis location now takes approximately 100 volunteer hours to get up and running at other locations. Additionally, since Sling Health's nonprofit program operates with pro bono mentorship and advising and a volunteer, trainee-led executive structure, as well as shared overhead costs nationally for economies of scale, the program operates at under $\$ 250,000$ in annual expenses across all 11 current chapters. This is substantially less than the cost of one faculty member per site and equates to costing under $\$ 850$ per student participant for a longitudinal medical innovation experience.

In addition to receiving direct network support with chapter formation and operations, the program's national scale enables Sling Health chapters and teams to access resources that are often not available on the local level. Sling Health and the AMA developed a Clinical Problem Database that enables healthcare professionals around the country to submit problems to the program. This resource provides a fertile field of topics for Sling Health teams to pursue, as well as an opportunity for clinicians outside of academic roles to advise trainees and engage with developing medical technology. The AMA also supports Sling Health chapter development via connections to medical schools, seed grants and other support to improve medical entrepreneurial training opportunities nationwide, not just in major tech-centered hubs. Sling Health continuously hones its curriculum with guidance from past teams and partner institutions to deliver entrepreneurial education more effectively. Sling Health also partners with Veterans Affairs Innovation Centers to facilitate work on technologies for veteran-focused clinical issues by providing mentorship, a pathway to study new technologies and a funding pathway for successful initiatives to grow. Strong pro bono legal support from Husch Blackwell enables teams to receive assistance with intellectual property, as well as corporate creation, as technologies begin to commercialize. These partnerships help
Sling Health provide access to high-level resources to chapters immediately upon founding. Partnerships with academic institutions across Sling Health's network also enable teams to connect with potential talent, mentors, investors, and clinical trial sites outside their home institution.

At the end of the year, Sling Health's National Demo Day brings together the top teams and partners from across the United States. By increasing the number of teams from a single site to a national scale, Sling Health's new technology pipeline draws national investors to expand opportunities for teams. Demo Day enables publicity and funding opportunities for budding companies, facilitates recruitment of more team members, enables collaboration on curricular development between partner sites, and allows chapter leadership to directly work with partner sites and determine the best ways to interface with and augment local ecosystems. Through these efforts, Sling Health has now expanded to 11 chapters across 15 universities, with expected growth by 10 more chapters over the next 5 years on the basis of current new-chapter interest and historical growth rates (Fig. 2). 
a

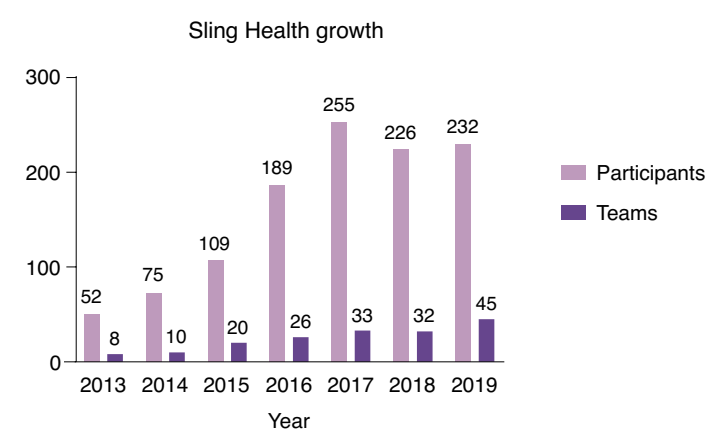

Race/ethnic background

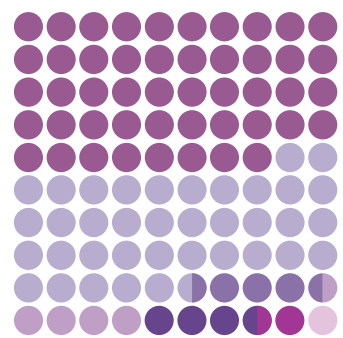

b

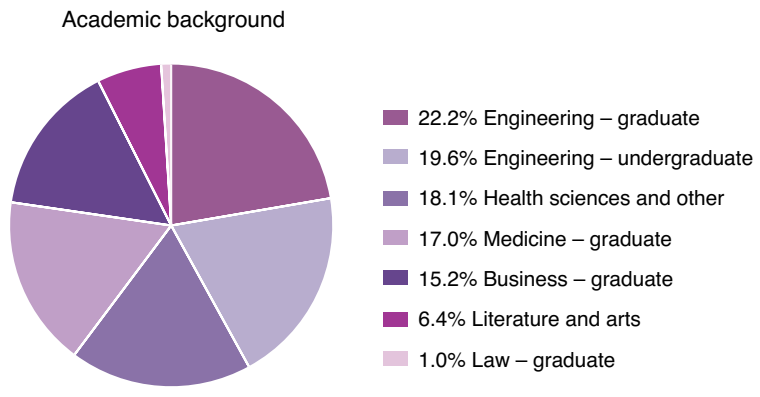

d

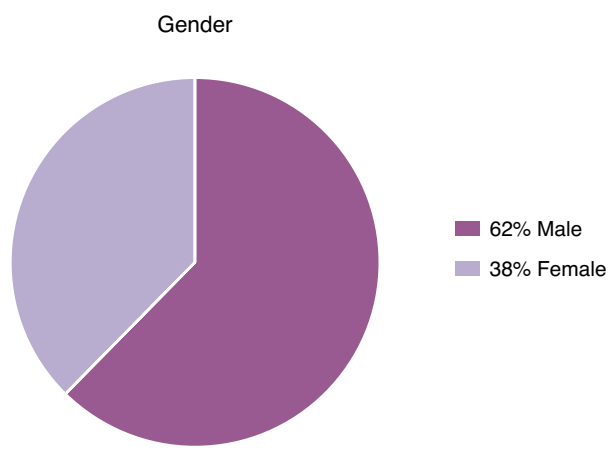

Fig. 3 | Enrollment in Sling Health and student distribution. a, Number of participants and teams in Sling Health network from 2013 to $2019 . \mathbf{b}-\mathbf{d}$, Sample of distribution of members by discipline, race and gender from 2016-2019 (St. Louis and Ann Arbor chapter data).

\section{Impact and conclusions}

Since forming in 2013, Sling Health has trained over 1,000 students nationally at 15 different universities to become better problem identifiers and solvers in healthcare. Team members span a wide variety of disciplines (Fig. 3b), enabling students to teach each other throughout their projects. Many students do not otherwise have the resources or opportunities to engage with entrepreneurship alone. Sling Health represents the first entrepreneurial experience for $70 \%$ of participants.

Sixty-three percent of members report that Sling Health influenced their career plans and choices, while $94 \%$ of members state the program offered medical technology exposure relevant to their professional goals. Membership to date has skewed toward male, white and Asian participants (Fig. 3c,d). Sling Health is aligning partnerships across academia and industry to address this participant disparity and build a more diverse, equitable and inclusive program for student participants, mentors and advisors. This growth area promises to strengthen our teams and enable a more diverse participant base to engage in medical entrepreneurship through Sling Health.

By engaging students early in their careers, Sling Health enables education and entrepreneurial involvement in a relatively low-risk atmosphere where it will not jeopardize a student's career path but will empower them to interface with medical technology development for life. Given that roughly 15 to $25 \%$ of trainees are medical students or residents (depending on chapter location), Sling Heath is actively training a generation of physicians to critically assess the status quo and address gaps with medical technology in order to advance patient care.

As it is a student-run nonprofit organization, Sling Health's local and national leadership boards also provide opportunities for trainees to interface with many developing medical technologies at once and enhance their entrepreneurial and managerial skills. In addition to trainees, many mentors anecdotally note that working with Sling Health improves their awareness of medical innovation and entrepreneurship. Sling Health's engagement of practicing physicians in particular connects doctors with clinical problems and knowledge to motivated students with the skills and time to develop new solutions. A small portion of members opt to pursue their Sling Health startups full time after the program, as most pursue further education or enter the workforce in a variety of roles. Regardless of career plans, the program provides experiential training for all members to communicate effectively across disciplines, assess clinical and market needs, analyze technical opportunities and limitations, iterate on prototypes and develop commercial implementation strategies to move ideas closer to helping patients.

Despite a plethora of minds interested in medical technology, the disconnect between disciplines and schools at large academic centers stifles innovation. Sling Health's platform serves as a bridge between the different silos of resources that exist at any given university and integrates these resources to drive student learning and medical innovation. Student-run and faculty-guided chapters allow the organization to adapt to the unique circumstances and needs of the academic center. The sheer number of clinicians and students at these centers forming teams, developing prototypes and forming startups through Sling Health demonstrates that the program is addressing these needs.

By empowering Sling Health participants to perform clinical need-finding via direct patient and clinician interaction, Sling Health produces a grassroots pool of clinical problems that stem directly from the patient. This democratization of clinical problem sourcing identifies problems that are both primary concerns for patients and entrepreneurially viable. The success of the Sling Health model has started to become visible in patient care as startups formed through the program continue to develop. Sling Health student teams have 
developed over 30 pending patents, over 25 startups and over $\$ 18$ million in investment in alumni companies, with clinical trials reaching over 80,000 patients. Sling Health's integration into academic centers enables teams to design and run clinical trials locally while our national model facilitates multi-university connections for growth. More and more prototypes and medical technologies are being developed each year by an untapped pool of students and doctors. This growing ecosystem offers the opportunity to spark new medical technologies and change healthcare both through the program directly and indirectly throughout Sling Health trainees' careers.

Sling Health's cost-effective, experiential training platform supports medical technology development across several clinical problem and solution domains, including digital health and health information technology, medical devices, diagnostic screening and global health technologies. Examples of projects supported in each domain include the following:

Digital health. After noticing stark inadequacy achieving hemoglobin A1c (HbAlc) goals for patients with diabetes mellitus in low-resource settings, a Sling Health team developed and launched a digital health intervention using automated text messages ${ }^{6}$ that led to an average $1.17 \%$ point reduction in $\mathrm{HbAlc}$ over four months in patients with baseline $\mathrm{HbAlc}>8 \%$ in a randomized, controlled trial ${ }^{7}$. This startup company has now implemented this intervention with thousands of patients and developed disease-specific automated interventions for over 20 diseases.

Medical devices. Sling Health has supported a wide range of medical devices, from improved endoscopic equipment to mobility support devices to laparoscopic sutures with memory material that simplify tying.

Diagnostic screening. After recognizing the difficulty getting patients to comply with colonoscopy screening for colorectal cancers and high-risk adenomas, a team developed a higher sensitivity, cost-effective stool screening platform based on stool-derived eukaryotic RNA ${ }^{8}$.

Global health. A team with family in Nigeria observed high death rates from neglected tropical infectious diseases such as schistosomiasis, so they developed a point-of-care DNA amplification device with a culturally tested user interface to achieve early diagnosis of the infection and enable faster treatment.

These sectors align with Sling Health's diversity of trainee expertise levels and focused initial prototype funds ranging from roughly $\$ 500$ to $\$ 2,500$, with further support available nationally. The program is unable to support drug development projects as they require more specialized education, resources and increased funds to study and pilot.

As evidenced here, this experiential, interdisciplinary approach balances the dual missions of training the next generation of medical innovators and advancing technologies with the potential to improve patients' treatments and lives. We have a responsibility to provide trainees insight into the medical technology development process and the ability to engage that process thoughtfully to advance care for our patients. Sling Health aims to carefully balance private entrepreneurial interest, which is foreign to most medical training but necessary for implementing new medical technologies, with professional and technical development, service to patients, teaching and leadership through careful curricular decisions and mentor and speaker selection, guided by local advisory boards. Overall, by training students in medical technology research, development and strategies for commercial, regulatory and clinical implementation, Sling Health helps members experience and understand the motivations, needs and interplay among the many aspects of the medical technology ecosystem. This type of training is fundamental for participants to accelerate the translation of research into better patient care, especially during these challenging times with COVID-19 requiring new levels of ingenuity and problem-solving in healthcare.

Sling Health is actively expanding educational programming, partnerships with healthcare organizations and national infrastructure in order to enhance support for student innovators and tailor services to evolving needs. Amid the COVID-19 pandemic, Sling Health is leveraging its experience and network to provide an accelerated one-month virtual boot camp, enabling over 100 students and mentors nationally and internationally to address new healthcare problems arising from the pandemic. The program is also building mechanisms to increase crosstalk between chapters for mentors and team talent sourcing, as well as provide support for teams that have progressed into early company stages. Sling Health plans to strategically grow connections between more universities and entrepreneurial ecosystems to further facilitate medical technology development. Overall, this experience navigating multiple university, entrepreneurial and legal systems to create a student-driven, collaborative initiative can serve as a guideline to efficiently develop similar systems elsewhere, affecting medical education, innovation and commercialization.

Stephen W. Linderman (D) 1,2,3凶 Abhinav J. Appukutty (D) ${ }^{4}$, Mario V. Russo ${ }^{5}$, Aadit P. Shah ${ }^{3}$ and Kavon Javaherian 2,6,7 ${ }^{1}$ Department of Medicine, Emory University, Atlanta, GA, USA. ${ }^{2}$ Washington University in St. Louis School of Medicine, St. Louis, MO, USA. ${ }^{3}$ Washington University in St. Louis School of Engineering and Applied Sciences, St. Louis, MO, USA. ${ }^{4}$ University of Michigan Medical School, Ann Arbor, MI, USA. ${ }^{5}$ Harvard Medical School, Boston, MA, USA. ${ }^{6}$ Olin Business School, Washington University in St. Louis, St. Louis, MO, USA. ${ }^{7}$ Department of Medicine, University of California San Francisco, San Francisco, CA, USA.

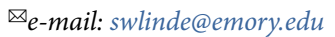

Published online: 5 October 2020 https://doi.org/10.1038/s41587-020-0689-7

References

1. Som, A., Charanya, T., Linderman, S. W. \& Siegel, J. S. Nat. Biotechnol. 32, 1063-1065 (2014).

2. Yock, P. G., Brinton, T. J. \& Zenios, S. A. Sci. Transl. Med. 3, $92 \mathrm{~cm} 18$ (2011).

3. Yazdi, Y. \& Acharya, S. Ann. Biomed. Eng. 41, 1822-1833 (2013).

4. Leuthardt, E. C. Neurosurgery 72 (Suppl. 1), 182-192 (2013).

5. Kurpinski, K., Johnson, T., Kumar, S., Desai, T. \& Li, S. Sci. Transl. Med. 6, 218fs2 (2014).

6. Peters, R. M. et al. JMIR Diabetes 2, e15 (2017).

7. Xu, R. et al. Telemed. J. E Health 26, 784-793 (2020).

8. Barnell, E. K. et al. Gastroenterology 157, 884-887.e3 (2019).

Acknowledgements

The authors would like to acknowledge all of Sling Health's student volunteers, faculty mentors and academic and industry sponsors. This program exists because of substantial student and volunteer support from thousands of individuals over the first seven years. We especially appreciate all of the institutional and individual support from Washington University in St. Louis, which enabled Sling Health's first chapter to grow and thrive since its infancy.

Author contributions

S.W.L., A.J.A, M.V.R., A.P.S. and K.J. serve as trainee volunteers on the board of the Sling Health National Network. S.W.L., A.J.A. and M.V.R. wrote the manuscript and all authors contributed to revision.

Competing interests

The authors declare no competing interests. 